\title{
Plan para la formación curricular de cátedras de accesibilidad en las facultades de Arquitectura en Colombia
}

\author{
Plan for curriculum training in subjects of accessibility in the Architecture \\ programs in Colombia
}

\author{
Leonardo Enrique Díaz Suárez \\ Arquitecto \\ Máster en Accesibilidad para Smart \\ City, la Ciudad Global. \\ Universidad Santo Tomás Seccional Bucaramanga \\ Correo electrónico: leonardoediaz@gmail.com
}

Recibido: 3 de mayo de 2016; aprobado: 7 de junio de 2016

\section{Cómo citar este artículo}

Díaz, L.E. (2016). Plan para la formación curricular de cátedras de accesibilidad en las facultades de Arquitectura en Colombia. Espiral, Revista de Docencia e Investigación, 6(1), 91- 106.

\section{Resumen}

Objetivo y tipología textual. El objetivo de este artículo de investigación, producto del trabajo final de maestría realizado por el autor, titulado "Protocolo para la formación curricular de cátedras de accesibilidad en las facultades de arquitectura en Colombia", es presentar una propuesta de trabajo que incluya la formación curricular de una cátedra de accesibilidad, mediante la configuración de un plan sistemático y flexible en el proceso formativo para proyectar la transformación del territorio mediante el desarrollo de un medio físico accesible que contribuya al respeto por los derechos humanos y la igualdad de oportunidades.

Metodología. En relación con lo anterior, este artículo de investigación se ha elaborado y sustentado a partir de un proceso metodológico cualitativo, compuesto por una fase de recopilación de la información sobre los diversos ámbitos de la accesibilidad y su relación con la academia, complementada por una fase de análisis donde se consignan todos los resultados obtenidos en los datos recibidos, con el fin de determinar la fase de síntesis que define el plan de la propuesta, para finalmente concluir con unas consideraciones sugerentes como ejes de ruta para la puesta en marcha de la cátedra.

Resultados y conclusión. Las condiciones de accesibilidad que históricamente han presentado los entornos urbanos y rurales han sido de un constante estado de exclusión y discriminación hacia las personas con discapacidad, ya sea de carácter temporal o permanente, o hacia los grupos poblacionales más vulnerables. Esto debido principalmente a los obstáculos físicos que poseen las ciudades o actitudinales de la propia ciudadanía.

Palabras clave: Accesibilidad, arquitectura, cátedra, discapacidad, formación curricular, inclusión.

\begin{abstract}
The objective of this research paper as a product of the final work of the master by the author, entitled "Protocol for the training curriculum chairs of accessibility in the faculties of architecture in Colombia" is to present a work proposal that includes curricular formation of a chair of accessibility, by setting a systematic and flexible plan in the training process, to project the transformation of the territory by developing an accessible physical environment that contribute to respect for human rights and equal opportunities.

In connection with the above, this research paper has been developed and supported from a qualitative methodological process, made up by a phase of gathering information on the various fields of accessibility and its relationship with the academy, complemented by a phase of analysis where all the results of data collection are recorded, in order to determine the synthesis phase that defines the plan of the proposal. Finally, it concludes with some suggestive considerations as route center lines for the implementation of the subject.

Accessibility conditions, that urban and rural environments have historically presented, have been a constant state of exclusion and discrimination against people with disabilities, whether temporary or permanent, or at the most vulnerable population groups. This is mainly due to the physical obstacles that cities or attitudes have about citizenship.
\end{abstract}

Keywords: Accessibility, architecture, professorship, disability, curricular formation, inclusion. 


\section{Introducción}

Apuntar hacia este propósito significa que el ejercicio proyectual o constructivo del futuro arquitecto se direccione con la responsabilidad social que sobre él recae al momento de realizar un diseño arquitectónico o un planteamiento urbanístico, basados en una calidad espacial donde la funcionalidad y la usabilidad se sustenten mediante el confort y la autonomía, con base en la accesibilidad universal (AU) y el diseño para todos (DPT).

El presente artículo de investigación se fundamenta en el trabajo final de maestría (TFM) realizado por el autor en el 2015, titulado "Protocolo para la formación curricular de cátedras de accesibilidad en Colombia", para optar por el título de "Máster en Accesibilidad para Smart City; La Ciudad Global", llevado a cabo por la Universidad de Jaén en España. A partir de este trabajo, se hizo un énfasis especial en los contenidos que se presentan a continuación, con respecto a la propuesta de un plan docente de enseñanza-aprendizaje, basado en competencias que le permita a la academia la inclusión o adaptabilidad del mismo, para dar cumplimiento a la legislación y normativa actual vigente sobre discapacidad y accesibilidad a nivel nacional y global.

\section{Metodología}

En el desarrollo de la investigación se ha aplicado un enfoque cualitativo en el que el autor ha interactuado con los referentes académicos analizados, ya sea de modo presencial o a distancia, para conocer las estructuras curriculares en su totalidad, lo cual ha permitido consolidar un estudio a pequeña escala como método de medición desde la realidad empírica. Dicho enfoque está compuesto de tres fases, con la finalidad de establecer una propuesta sustentada que conduzca a la definición del plan para la implementación de la cátedra de accesibilidad en los programas de arquitectura del país que aún no la incluyen dentro de sus mallas curricu- lares o para la actualización de las ya existentes, conformado de la siguiente manera:

\section{Fase de recopilación de la información}

Se realizó a partir de una búsqueda de datos sobre fuentes bibliográficas primarias, secundarias y terciarias acerca de todas las temáticas necesarias para la conformación del marco teórico y conceptual, al mismo tiempo que se investiga en la legislación colombiana los artículos pertinentes para argumentar la inclusión de la cátedra de accesibilidad. Se identificaron las facultades de arquitectura en Colombia que actualmente incluyen la cátedra de accesibilidad con carácter obligatorio en sus programas curriculares, estableciendo contacto con sus respectivos directores, para obtener información y documentación correspondiente a los planes de asignatura desarrollados en cada una de ellas. Se detectó cuáles han sido las investigaciones afines con el tema, emprendidas en el territorio nacional, con el fin de demarcar puntos de partida y delimitantes para la cobertura y alcance de la investigación. Mediante las oportunas y acertadas tutorías académicas se logró configurar una estructuración de los contenidos por tratar, con los cuales se definieron los objetivos y proyecciones de análisis para la concreción de la siguiente fase.

\section{Fase de análisis}

Con toda la información organizada y consignada, se clarificaron los resultados obtenidos del proceso metodológico, conforme a los objetivos definidos, para dar paso a un espacio de discusión, donde se configuró un comparativo entre las cátedras de accesibilidad obligatorias en los programas de arquitectura del país, por medio de una estructura argumentativa que facilitó resaltar y valorar los puntos en común o diferencias entre ellas. Además de esto, se incorporó una cátedra de accesibilidad con carácter de obligatoriedad del contexto internacional, para contrastar lo implementado en el ámbito regional con lo desarrollado en el 
exterior, con la intención de enfocar de modo sistemático la finalidad de la investigación.

\section{Fase de síntesis}

Etapa final del proceso, en la cual se definió la configuración de un plan para la formación curricular de cátedras de accesibilidad en Colombia, que dé respuesta satisfactoria al objetivo general definido en el presente artículo. Junto a ello, se establecen las conclusiones con unas consideraciones sugerentes por seguir para la implementación de la cátedra, basadas en otros procesos afines adelantados satisfactoriamente en el país.

\section{Resultados}

\section{La necesidad de una cátedra de accesibilidad obligatoria en todas las facultades de arquitectura en Colombia}

La población con discapacidad en Colombia, según cifras del Censo General de 2005 realizado por el Departamento Administrativo Nacional de Estadística (DANE), correspondió a un total de 2624898 personas, equivalente al $6,3 \%$ de una población total nacional de 41468384 habitantes, DANE (2008). A partir de esta estadística, el Ministerio de Salud y Protección Social (MINSALUD) ha venido desarrollando la aplicación del Registro para la Localización y Caracterización de las Personas con Discapacidad (RLCPD), cuya cobertura nacional al 21 de octubre de 2013, ha sido de 1037534 habitantes, correspondiente al $2 \%$ de las personas identificadas en el 2005 con algún tipo de discapacidad, de acuerdo al Ministerio de Salud (2014) Observatorio Nacional de Discapacidad, Boletín 1, p.3.

Teniendo en cuenta lo anterior y en concordancia con la legislación establecida en el territorio colombiano, a través de las diversas leyes y decretos publicados para atender los asuntos sobre discapacidad y accesibilidad, se establece en el numeral 1 del artículo 14 sobre Acceso y Accesibilidad, promulgado por la Ley
Estatutaria 1618 de 2013, la ejecución de todos los ajustes razonables necesarios siguiendo los postulados del Diseño Universal, para garantizar el acceso al entorno físico, al transporte, a la información y las comunicaciones, el espacio público, los bienes públicos, los lugares abiertos al público y los servicios públicos, tanto en zonas urbanas como rurales, de manera que no se excluya a ninguna persona en razón de su discapacidad. Esto se emite para dar cumplimiento al artículo 9 de la Ley 1346 de 2009, la cual aprueba la Convención sobre los Derechos de las Personas con Discapacidad (CDPD), promulgada por la Organización de Naciones Unidas (ONU) desde el 2006.

En el mismo artículo 14 de la Ley 1618, en los numerales 3 , 4 y 5 se define lo siguiente:

3. Las entidades municipales y distritales, con el apoyo del Gobierno departamental y nacional, y respetando la autonomía de cada región, deberán diseñar, en un término no mayor a 1 año, un plan de adecuación de vías y espacios públicos, así como de accesibilidad al espacio público y a los bienes públicos de su circunscripción. En dicho plan deberán fijarse los ajustes razonables necesarios para avanzar progresivamente en la inclusión de las personas con discapacidad, establecer un presupuesto y un cronograma que, en no más de 10 años, permita avanzar en niveles de accesibilidad del $80 \%$ como mínimo. Dicho plan deberá fijar los criterios de diseño universal que deberán ser acatados en todas las obras públicas y privadas de la entidad pública a partir de su adopción.

4. Implementar las medidas apropiadas para identificar y eliminar los obstáculos y para asegurar la accesibilidad universal de todas las personas con discapacidad al ambiente construido, transporte, información y comunicación, incluyendo las tecnologías de información y comunicación y otros servicios, asegurando las condiciones para que las personas con discapacidad puedan vivir independientemente.

5. Dar efectivo cumplimiento a la normativa sobre accesibilidad en la construcción o adecuación de las obras que se ejecuten sobre el 
espacio público y privado, que presten servicios al público debiendo cumplir con los plazos señalados.

En resumen, la legislación es bien clara al establecer un plazo de diez años para ejecutar los ajustes necesarios en la adecuación de vías y espacios públicos, que permitan la inclusión de las Personas con Discapacidad, para alcanzar unos niveles mínimos de accesibilidad del $80 \%$.

Asimismo, la Ley 361 de 1997, en el artículo 8 determina:

Artículo $8^{\circ}$. El Gobierno a través del Ministerio de Educación Nacional tomará las medidas necesarias para que tanto en el proceso educativo como en el de culturización en general, se asegure dentro de la formación integral de la persona la prevención de aquellas condiciones generalmente causantes de limitación.

Para estos efectos las entidades públicas y privadas que tengan por objeto la formación y capacitación de profesionales de la educación, la salud, trabajadores sociales, psicólogos, arquitectos, ingenieros, o cualquier otra profesión que pueda tener injerencia en el tema, deberán incluir en sus currículos temáticas referentes a la atención y prevención de las enfermedades y demás causas de limitación y minusvalías.

Y en el parágrafo del artículo 47 referente a la eliminación de barreras arquitectónicas, se establece que:

Parágrafo. En todas las facultades de arquitectura, ingeniería y diseño de la República de Colombia se crearán talleres para los futuros profesionales de la arquitectura, los cuales serán evaluados y calificados con el objetivo primordial de fomentar la cultura de la eliminación de las barreras y limitaciones en la construcción.

Este panorama nos conduce a reflexionar sobre la necesidad de contar con profesionales capacitados para dar cumplimiento y ejecución a lo establecido en materia de discapacidad y accesibilidad en el territorio nacional. No obstante, la situación real del estado de avance para la implementación de los ajustes razonables al medio físico, está muy lejos de mostrar avances para cumplir con los plazos de adecuaciones estipulados. $Y$ esto sucede, ya sea por desconocimiento de la legislación (lo cual no constituye un argumento para su incumplimiento), por falta de conocimiento y aplicación de la normatividad dispuesta para tales fines, o por poca instrucción, difusión y fomento por parte de la academia, además de otras variables o barreras derivadas desde la gestión o desde la misma sociedad, que excluye con comportamientos actitudinales o de indiferencia.

Debido a ello, es de vital importancia que desde las instituciones de educación superior, específicamente para el caso de los programas de pregrado en arquitectura, se promueva la inclusión obligatoria de una cátedra de accesibilidad para la formación de los futuros profesionales del oficio, cuyo enfoque permita llevar a cabo desde el mismo momento del ejercicio proyectual, la aplicación transversal de todos los criterios y parámetros de Accesibilidad Universal aprendidos y puestos en práctica a partir de las competencias adquiridas en las aulas de clase, para incorporar progresivamente o adecuar objetos arquitectónicos y zonas urbanas o rurales acordes con los principios del Diseño Universal (DU), que contribuyan a la configuración de ciudades más humanas para todos.

\section{Cátedras de accesibilidad con carácter de obligatoriedad}

En el proceso de recopilación de datos para identificar el número de facultades de arquitectura en el territorio nacional, se detectó que para el 2014, según el Sistema Nacional de Información de Educación Superior (SNIES) en Colombia se encontraban registrados 58 programas presenciales de arquitectura activos (Castellanos, 2015), repartidos en todo el territorio nacional. En estos programas se comprobó que tan solo tres de ellos cuentan con una cátedra de accesibilidad con carácter 
obligatorio y continuo, denominadas como se describe seguidamente:

De las tres asignaturas valoradas, se aprecia que tan solo la UAC aborda la temática bajo la modalidad de taller práctico, mientras que la otras dos lo abordan como materia teórica.

Se han desarrollado otros proyectos a nivel nacional como el denominado "Maxiválidos", Becerra (2007), creado y dirigido por el diseñador industrial Ricardo Becerra Sáenz en el 2006, e implementado por la Facultad de Arquitectura y Diseño de la Pontificia Universidad Javeriana de Bogotá, aunque en la actualidad ya no tiene continuidad, según lo manifestado por su creador.

Posterior a ello, la Universidad Nacional de Colombia con sede en Medellín crea la práctica académica especial titulada "Accesibilidad e Inclusión por Vía de Entorno".

Castellanos (2015), Accesibilidad Universal en todos los programas de arquitectura de universidades colombianas (p. 84), dirigida por el arquitecto Jorge Hernando Torres Holguín y desarrollada en el segundo semestre de 2013 y primer semestre de 2014, con carácter de libre elección y sin continuidad en la actualidad.

Otro proyecto similar fue el desarrollado por la Universidad Nacional de Colombia, sede en Bogotá, titulado "Diseño Inclusivo, Cátedra de sede Jorge Eliécer Gaitán" (Fernández \& Sarmiento, 2015) en el primer semestre del año en curso, coordinado por la terapeuta ocupacional Aleida Fernández y la diseñadora industrial Martha Patricia Sarmiento. Sin embargo, ha sido una cátedra de libre elección y tampoco mantuvo la continuidad hasta la fecha.

De igual manera, se incluyó en el análisis una asignatura afín con el tema y de carácter obligatorio a nivel internacional, para lo cual se confirmó que la Universidad Internacional de Cataluña (UIC) ubicada en Barcelona, España, cuenta con una materia denominada Cátedra de Accesibilidad, dirigida por el arquitecto Enrique Rovira-Beleta Cuyás, con duración de un semestre e impartida en el tercer año del plan de estudios.

Tabla 1. Cátedras de accesibilidad con carácter de obligatoriedad en Colombia

\begin{tabular}{|c|c|}
\hline Asignatura & Universidad \\
\hline $\begin{array}{l}\text { Taller de Arquitectura Incluyente. } \\
\text { Director: Arquitecto Alfredo de Jesús Arrieta Príncipe } \\
\text { Semestre: VIII }\end{array}$ & $\begin{array}{l}\text { Universidad Autónoma del Caribe } \\
\text { (UAC) }\end{array}$ \\
\hline $\begin{array}{c}\text { Cátedra de Accesibilidad Universal. } \\
\text { Directora: Arquitecta Gilda Marina Toro Prada } \\
\text { Semestre: VII }\end{array}$ & Universidad de la Salle de Bogotá (UNISALLE) \\
\hline $\begin{array}{c}\text { Cátedra de Accesibilidad. } \\
\text { Directora: Psicóloga María del Pilar Pinzón Rueda } \\
\text { Semestre: VIII }\end{array}$ & $\begin{array}{l}\text { Universidad Santo Tomás, Bucaramanga } \\
\text { (USTABUCA) }\end{array}$ \\
\hline
\end{tabular}

Fuente: Elaborado por el autor a partir de consultas con los respectivos directores de cada asignatura. 
Tabla 2. Componentes fundamentales del plan docente por competencias para una asignatura

Esta fase tiene como propósito ubicar al estudiante dentro del proceso de formación en la asignatura. Para ello se indica el nombre del Plan Docente, y se identifica la institución, la facultad y el programa en el cual se va a llevar a cabo. Así mismo, se indica de forma resumida el número de créditos, la competencia o competencias por formar, y el nombre del docente o docentes a cargo.
El propósito de este componente consiste en describir la ruta general del proceso de formación que se va a seguir en la asignatura.

Para ello se describen con detalle las competencias por formar y los créditos.
3. Planeación de talleres y productos

Con el fin de desarrollar las competencias propuestas en el apartado anterior, se hace una planeación de talleres, indicándose el producto para obtener en cada uno de ellos y las fechas tentativas de realización.
Se describen en general las pautas y estrategias para evaluar las competencias. Se enfatiza en los indicadores y en las evidencias más importantes, así como también en los niveles de logro de los indicadores.
5. Competencias docentes

Se describen las principales competencias genéricas y específicas que deben tener los docentes que trabajarán en la asignatura. Esto debe ser acorde con la filosofía institucional y la orientación del programa.

\section{Desarrollo de los talleres}

En esta parte se desarrollan con detalle los talleres descritos en la parte 3 del Plan Docente, tal y como se van a trabajar con los estudiantes. Es importante indicar que cada taller busca contribuir al desarrollo de las competencias propuestas, y por lo tanto deben estar articulados entre sí.

Fuente: Tejada et al., 2006, p.104

\section{Estructura argumentativa para el comparativo entre los syllabus de las cátedras de accesibilidad analizadas}

Con la intención por explorar y analizar semejanzas y diferencias, así como las fortalezas y debilidades entre las cuatro cátedras de accesibilidad de carácter obligatorio detectadas para el análisis, se hizo necesario establecer una estructura argumentativa que permitiese ser aplicada a cada uno de los syllabus en el proceso de análisis conducente al esclarecimiento y formulación del planteamiento definido en el objetivo general de la investigación. Por consiguiente, se implementó la estructura argumen- tativa expuesta por Tejada et al. (2006), compuesta por seis componentes fundamentales para definir el plan docente de una asignatura, en el siguiente orden:

Del comparativo realizado a las asignaturas consignadas en la tabla 1, incluida la Cátedra de Accesibilidad de la UIC, se pudo apreciar como punto en común para los cuatro casos, que no se tuvo en cuenta el tema de las competencias docentes. Por otra parte, el número de créditos académicos para las cátedras oscila entre 2 y 2.5 , siendo este último, el correspondiente a la cátedra internacional. Contrario a ello, el taller de la UAC cuenta con un número de cuatro cré- 


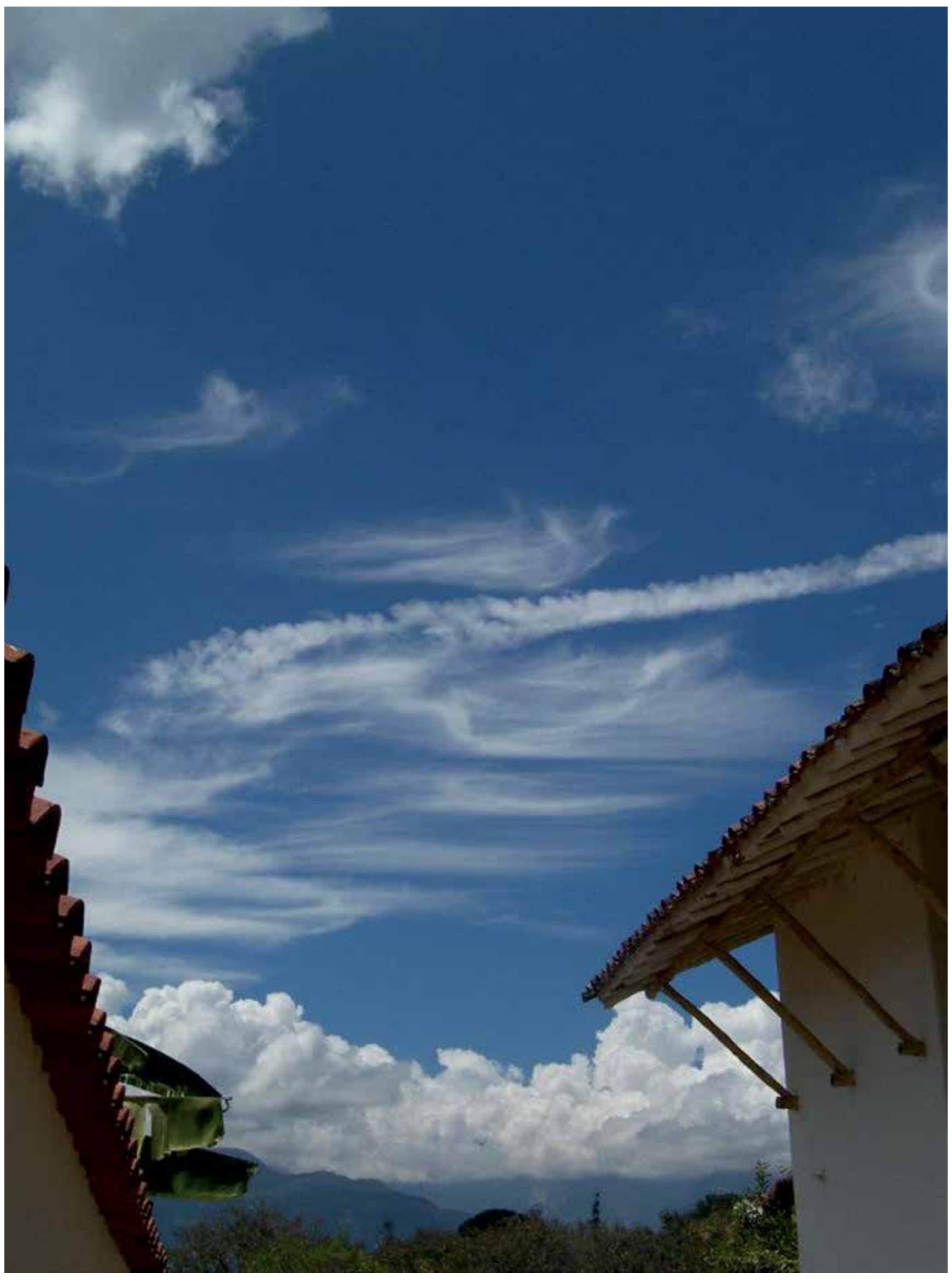


ditos académicos, por ser el único en el análisis de modalidad práctica. A su vez, se observa que en los contenidos del taller, al no tener una modalidad teórica, no se pueden incluir con mayor cobertura las temáticas que deberían contemplarse en el desarrollo de una cátedra de accesibilidad, abordándose como un tema complementario y no como el asunto central del curso, debido al énfasis propio de dicha asignatura que debe estar enfocado finalmente al diseño de un objeto arquitectónico. Por tal razón, se considera que lo ideal para la implementación del ámbito de la accesibilidad, ha de ser a partir de cátedras teórico-prácticas en las cuales se pueda tratar con mayor extensión los contenidos pertinentes sobre el tema y de esta manera consolidar el aprendizaje en el estudiante de modo más completo, permitiendo con ello que se logre su aplicabilidad para alcanzar la transversalidad de modo integral en las demás asignaturas de la malla curricular, como son el taller de diseño, urbanismo, construcción, expresión, metodología de la investigación, proyecto de grado, entre otros.

La Cátedra de Accesibilidad de la USTABUCA se enmarca dentro del área de curso electivo profesional ofrecida para los alumnos de octavo semestre. Sin embargo, la asignatura se enseña con carácter de obligatoriedad para todos los estudiantes que estén cursando dicho nivel, llegando a un total de cuatro salones con veinte estudiantes en promedio por clase, para el año en curso'.

Ante la disparidad observada en la designación cronológica dentro del currículo, se sugiere que la ubicación de una cátedra de accesibilidad en un programa de arquitectura, ha de ser a mitad de carrera, ya sea en quinto semestre o en tercer año de estudios, tal como lo establece la asignatura de la UIC, puesto que se considera un nivel de formación profesional en el cual se empiezan a implementar temas de gran importancia y complejidad para el ejercicio proyec-

1 Dato aportado por el autor con base en el trabajo realizado como docente a cargo de la cátedra en mención durante el 2015. tual, en donde la pertinencia de la accesibilidad se hace muy oportuna para su aplicabilidad en un mayor número de proyectos académicos; estando en el punto intermedio del proceso formativo en el que ya se desarrolla un sentido crítico y de conocimiento sobre las necesidades del entorno físico. No es conveniente que el estudio y aplicación de la accesibilidad se haga presente tan solo hasta finalizar el cuarto año de carrera profesional.

\section{Plan para la formación curricular de cátedras de accesibilidad en las facultades de arquitectura en Colombia}

La fase final de la presente investigación define la configuración del plan que le permita a las facultades de arquitectura del país la facilidad de crear e implementar en sus currículos generales, una cátedra de accesibilidad claramente configurada para contribuir a la formación integral del arquitecto.

A continuación se desglosa la estructura sistemática, con base en los componentes consignados en la tabla 2, cuya aplicabilidad ha permitido concretar esta propuesta:

\section{Planeación de aspectos formales}

Nombre de la asignatura: Accesibilidad Universal

Programa: Arquitectura

Semestre: Quinto o tercer año de estudios

Modalidad: Teórico - práctica

Tipo: Obligatorio

Créditos: 2

\section{Semanas: 16}

Objetivo general: Crear un espacio académico para la reflexión, sensibilización y concientización sobre las diversas capacidades humanas y su accesibilidad a los entornos urbanos y rurales, que garantice la inclusión y la calidad de 
vida para todas las personas sin discriminación alguna, por motivos de discapacidad o vulnerabilidad.

Perfil de egreso: El estudiante estará es capacidad de aplicar todos los criterios de accesibilidad adquiridos en el diseño y ejecución de proyectos arquitectónicos y urbanos en cualquier etapa de su proceso académico y ejercicio profesional, de modo tal que difunda y promueva el compromiso social que debe aportar la arquitectura en la proyección y desarrollo del hábitat incluyente para toda la diversidad humana.

\section{Competencias y créditos}

Horas semanales: presenciales: 3 , trabajo independiente: 3

Total horas presenciales: 48

Total horas de trabajo independiente: 48

Total intensidad horaria: $96 \mathrm{hrs}$.

Número de créditos de la asignatura: 96/48 = 2

Competencias genéricas: Con base en el proyecto Tuning Latinoamérica (Beneitone et al., 2007).

- Capacidad de recopilar, procesar y analizar información de los diferentes tipos de fuentes bibliográficas para desarrollar proyectos de investigación.

- Actitud de compromiso con la calidad, respeto por la diversidad y responsabilidad social con el medio físico y cultural de la ciudadanía.

- Capacidad de aprendizaje y actualización constante sobre la temática de estudio y la profesión.

- Capacidad de sentido crítico y criterio propio para detectar y plantear la solución de problemas.
- Capacidad de autoevaluación para determinar fallos y aciertos en la formación profesional.

- Capacidad para ejecutar un trabajo de forma independiente, así como de conformación y participación en trabajos grupales.

- Conocimiento y destreza para aplicar apropiadamente el uso de las tecnologías de la información y las comunicaciones.

- Aptitud para expresar a través de las diversas vías de comunicación las ideas o propuestas a las demás personas.

- Capacidad de transmitir los conocimientos teóricos adquiridos, en la puesta en marcha del ejercicio académico y profesional.

- Actitud para el adecuado manejo de las relaciones interpersonales.

\section{Competencias específicas}

- Conocimiento de las diversas capacidades funcionales, en relación con la necesidad de inclusión social en igualdad de oportunidades.

- Conocimiento de criterios y parámetros del Diseño Universal para eliminar barreras arquitectónicas y ejecutar construcciones accesibles.

- Capacidad para aplicar la normatividad y legislación existente en proyectos de diseño arquitectónico y urbano o de nueva construcción.

- Capacidad para diagnosticar el nivel de accesibilidad en edificaciones públicas, privadas y entornos urbanos o rurales existentes.

- Capacidad para desarrollar propuestas de adecuación de espacios incluyentes en edificaciones públicas, privadas y entornos urbanos o rurales existentes. 
- Aptitud para adaptar y preservar el patrimonio construido de manera que pueda ser recorrido, valorado y estudiado por todas las personas.

- Capacidad para evaluar y adecuar los entornos naturales con soluciones de accesibilidad que no alteren el ecosistema y preserven el equilibrio ecológico del lugar.

- Capacidad para adaptar o modificar la vivienda para hacerla accesible y proyectarla como el hogar para toda la vida.

- Capacidad para implementar sistemas de evacuación en edificaciones y entornos habitables nuevos y existentes.

- Conocimiento de normatividad sobre accesibilidad y "Diseño para Todos" de carácter internacional para implementar la inclusión en diversos contextos.

\section{Planeación de talleres y productos}

Módulo 1. Diversidad humana e igualdad de oportunidades: El estudiante realiza un proceso de conocimiento reflexivo, de sensibilización y concientización sobre la diversidad funcional y la necesidad de aportar soluciones para incentivar la calidad de vida de las personas, en especial quienes posean alguna discapacidad, vulnerabilidad o víctimas del conflicto.

Módulo 2. Marco jurídico local, nacional e internacional sobre accesibilidad: Conocimiento de la legislación colombiana e internacional en materia de discapacidad y accesibilidad y su pertinencia en el ejercicio de la profesión.

Módulo 3. Introducción a la accesibilidad: Estudio y análisis de los principios del Diseño Universal y conceptos básicos de accesibilidad.

Módulo 4. Accesibilidad en el espacio urbano y transporte: El estudiante identifica los componentes del espacio público, de mobiliario urbano y su conexión con la movilidad y el transporte, evaluando la temática con la normativa existente. Se definen las consideraciones para el tratamiento del patrimonio construido y los entornos naturales.

Módulo 5. Accesibilidad en edificaciones de uso público: El estudiante comprende e interpreta la normativa de accesibilidad para edificios de uso público y su aplicación para diagnosticar y proponer soluciones en intervenciones de tipo arquitectónico o constructivo.

Módulo 6. Accesibilidad en edificaciones residenciales, la vivienda accesible: Conocimiento, interpretación y aplicación de la normativa para acceder a edificios residenciales. Soluciones de adaptabilidad, usabilidad y domótica para configurar las condiciones óptimas de accesibilidad en la vivienda.

\section{Proceso de evaluación}

Criterios: Se ha de orientar con la intención de adquirir las competencias estipuladas en la configuración de la cátedra de accesibilidad, enfocándose en las capacidades del alumnado para identificar, investigar, diagnosticar, analizar, evaluar y desarrollar propuestas para eliminar las barreras arquitectónicas que presentan los entornos urbanos y rurales. Para ello, se sugieren los siguientes ejercicios teórico-prácticos para la aprobación de cada uno de los módulos propuestos:

Módulo 1. (Práctico). Realización de un trabajo de campo grupal en el que los estudiantes asumirán la diversidad funcional como propia, en un recorrido urbano que permita identificar los problemas de accesibilidad presentes en el medio físico, de donde se obtendrá el material audiovisual para analizar, sensibilizar y concientizar sobre las barreras arquitectónicas y actitudinales que genera la sociedad.

Módulo 2. (Teórico). Comparativo entre la Ley 361 de 1997 en Colombia y la Ley Estatutaria 1618 de 2013 en Colombia, a partir de una estructura argumentativa que enfatice la finalidad, los principios, los componentes, las enmiendas y la vinculación con otros tratados que promulgue cada una de ellas. 
Módulo 3. (Teórico). Resumen crítico acerca de los conceptos básicos de la accesibilidad y los principios del Diseño Universal argumentados con ejemplos de buenas y malas prácticas a nivel local sobre el tema.

Módulo 4. (Práctico). Ejecución de una auditoría de accesibilidad a un espacio público de la ciudad, en la cual se evalúe el estado del itinerario peatonal y los diversos elementos de mobiliario que componen el entorno analizado, presentando propuestas de diseño para solucionar los problemas existentes.

Módulo 5. (Práctico). Desarrollo de una cadena de accesibilidad en un edificio de uso público de la ciudad, efectuando el diagnóstico y propuestas a partir de ajustes razonables, aplicando los parámetros establecidos por norma.

Módulo 6. (Práctico). Diagnóstico y propuesta de adecuación de la vivienda de cada estudiante, con el objetivo de hacerla accesible para un adulto mayor usuario de silla de ruedas o para una persona con discapacidad visual o auditiva.

Evaluación final. (Teórico-práctico). El estudiante deberá entregar un informe técnico acerca de los criterios de accesibilidad estudiados a lo largo de la cátedra y su implementación en el proyecto arquitectónico o urbano llevado a cabo en el respectivo taller de diseño que esté cursando durante el semestre. Se podrá alternar semestralmente con la ejecución de un taller práctico enfocado al diseño de mobiliario urbano, tal como el diseño de una parada de autobús accesible, ente otros, donde se apliquen conceptos de Diseño Universal, ergonomía y domótica, con la finalidad de ser presentado en una exposición final abierta al público.

\section{Competencias docentes}

Competencias genéricas:

- Conocimiento de las tecnologías de la información y las comunicaciones (TIC) para su aplicación en los procesos de enseñanza-aprendizaje.

- Actitud de compromiso y responsabilidad social con la diversidad humana.

- Capacidad para el dominio de nuevas modalidades de pedagogía, ya sean presenciales o virtuales.

- Actitud para valorar equitativamente la libre opinión del estudiante.

- Capacidad para comprender y analizar los contextos locales e internacionales, así como la multiculturalidad.

- Capacidad para ejercer la docencia y el liderazgo sin discriminación de ningún tipo y con la misma calidad tanto en grupos pequeños como más numerosos (ponencias).

- Capacidad para autoevaluar y modificar los procesos de enseñanza-aprendizaje.

- Capacidad para ejecutar y promover el trabajo en equipo y la cooperación entre los estudiantes.

- Capacidad de discurso y terminología propia de la materia.

- Actitud de respeto y comprensión hacia el alumno.

- Capacidad para evaluar el aprendizaje del estudiante con honestidad, justicia y puntualidad.

- Capacidad para desarrollar metodologías y estrategias creativas, innovadoras y motivacionales que beneficien el aprendizaje y la formación integral del futuro profesional en el campo laboral e interpersonal.

Competencias específicas:

- Conocimiento especializado y actualizado sobre el ámbito de la accesibilidad y el 
Diseño para Todos que garantice la validación de los contenidos que se difunden.

- Capacidad para aplicar soluciones adquiridas a través de la experiencia a problemas propios de la materia y afines con ella, derivados de los cuestionamientos o dudas presentadas por el alumno.

- Capacidad para seleccionar el material de curso adecuado y actividades pertinentes para generar la sensibilización y concientización que ha de adquirir el estudiante para comprender la diversidad funcional.

- Capacidad para propiciar la interacción, la retroalimentación y el debate en cuanto a los temas de la accesibilidad que demandan análisis y puntos de vista diferentes, para la búsqueda de soluciones consensuadas e incluyentes.

- Conocimiento para asesorar o liderar proyectos de investigación relacionados con el diagnóstico, diseño y propuesta de soluciones accesibles para los entornos urbanos o rurales construidos o de nueva construcción.

- Capacidad para incorporar los ámbitos de la Accesibilidad Universal de manera transversal en todas las disciplinas y delimitantes que requiere el oficio de hacer arquitectura.

- Conocimiento de la evolución y transformación de las ciudades y la planificación del territorio, como respuesta a los nuevos criterios de sostenibilidad y accesibilidad que plantean las Smart cities.

\section{Desarrollo de los talleres}

La metodología para desarrollar la ejecución semanal de cada clase se plantea de la siguiente manera:

- Semana 1. Diversidad humana e igualdad de oportunidades.
Contenidos: Concepto de diversidad humana, diversidad funcional y tipos, estereotipos, datos demográficos, modelos históricos de la discapacidad, Clasificación Internacional del Funcionamiento, de la Discapacidad y de la Salud (CIF), productos de apoyo, ciudades inclusivas, planteamiento ejercicio de evaluación módulo 1.

Actividades: Clase magistral, conversatorio.

- Semana 2. Diversidad humana e igualdad de oportunidades.

Contenidos: Desarrollo del ejercicio de evaluación módulo 1.

Actividades: Experiencia vivencial, exposiciones, debate, puesta en común, socialización.

- Semana 3. Marco jurídico nacional, local e internacional sobre accesibilidad.

Contenidos: Legislación colombiana, Convención sobre los derechos de las personas con discapacidad, legislación local, planteamiento ejercicio de evaluación módulo 2.

Actividades: Trabajo en equipo, retroalimentación de lecturas, conversatorio, puesta en común, elaboración de textos.

- Semana 4. Introducción a la accesibilidad.

Contenidos: Concepto de Accesibilidad Universal y Diseño para Todos, Principios del Diseño Universal, criterios DALCO (Deambulación, aprehensión, localización y comunicación), usabilidad, ergonomía, antropometría, diseño inclusivo, cadenas de accesibilidad, planteamiento ejercicio de evaluación módulo 3.

Actividades: Clase magistral, conversatorio, retroalimentación de lecturas. 


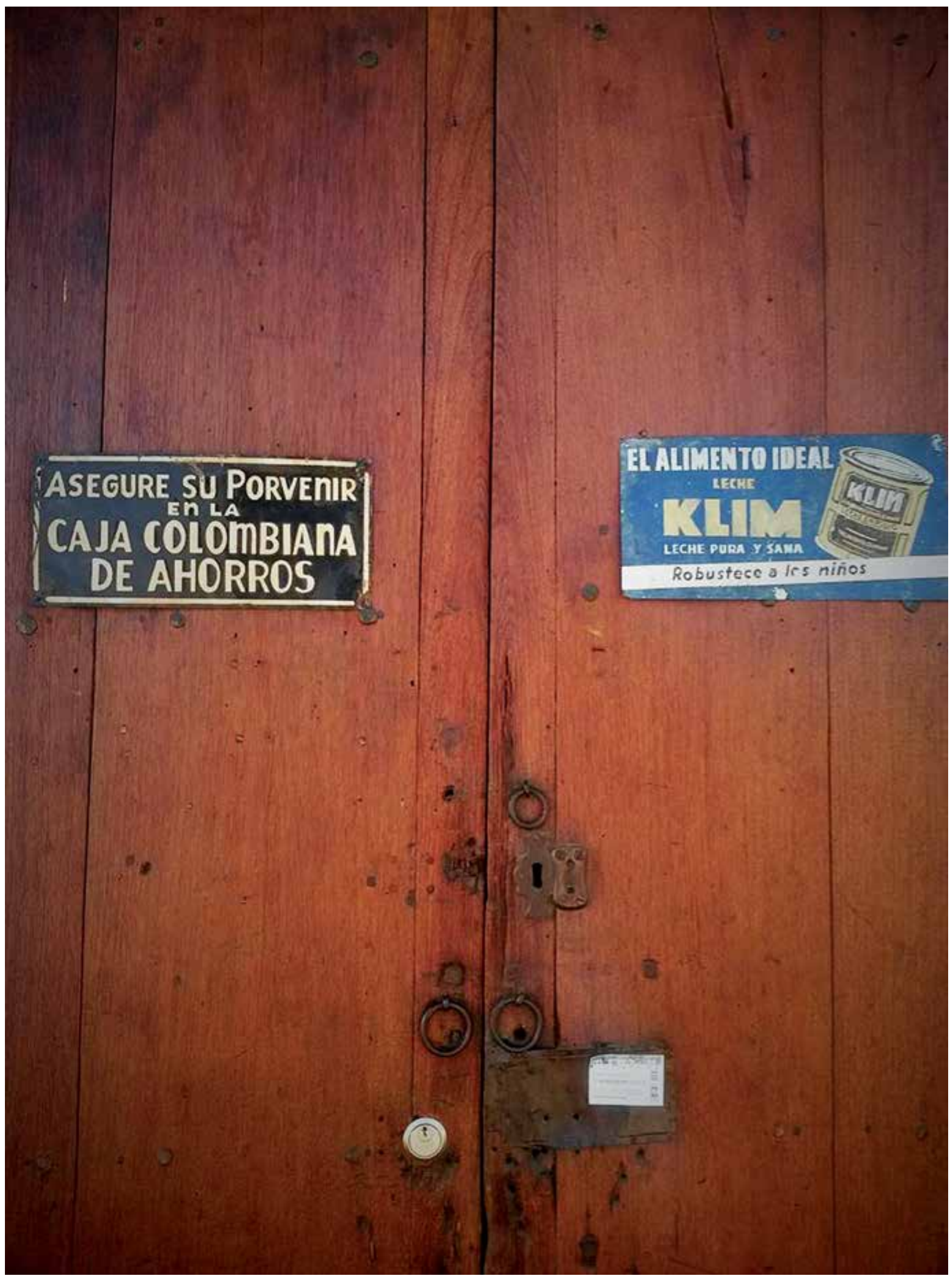


- Semana 5. Accesibilidad en el espacio urbano y transporte.

Contenidos: Itinerarios peatonales, cambios de nivel, cruces peatonales y tipos, elementos de urbanización y mobiliario urbano, planteamiento del ejercicio de evaluación módulo 4.

Actividades: Trabajo en equipo, retroalimentación de lecturas, análisis normativo, elaboración de textos comparativos, conversatorio.

- Semana 6. Accesibilidad en el espacio urbano y transporte.

Contenidos: Accesibilidad en el Sistema Integrado de Transporte Masivo, tipos de transporte, accesibilidad en el patrimonio construido, accesibilidad en entornos naturales, buenas y malas prácticas de accesibilidad.

Actividades: Clase magistral, conversatorio, debate.

- Semana 7. Accesibilidad en el espacio urbano y transporte.

Contenidos: Revisión y seguimiento del ejercicio de evaluación módulo 4.

Actividades: Trabajo de campo, trabajo en equipo, estudio de casos, retroalimentación de lecturas, aplicación normativa, asesoría de diseño, elaboración de textos e infografías.

- Semana 8. Accesibilidad en edificaciones de uso público.

Contenidos: Parqueaderos accesibles, accesos a la edificación, circulación horizontal, circulación vertical, evacuación accesible, planteamiento del ejercicio de evaluación módulo 5.
Actividades: Exposiciones, conversatorio, debate.

- Semana 9. Accesibilidad en edificaciones de uso público.

Contenidos: Baterías sanitarias, edificios de concurrencia pública, mobiliario, señalización, wayfinding, comunicación y orientación, buenas y malas prácticas de accesibilidad.

Actividades: Exposiciones, conversatorio, debate.

- Semana 10. Accesibilidad en edificaciones de uso público.

Contenidos: Revisión y seguimiento del ejercicio de evaluación módulo 5.

Actividades: Trabajo de campo, trabajo en equipo, estudio de casos, retroalimentación de lecturas, aplicación normativa, elaboración de textos e infografías.

- Semana 11. Accesibilidad en edificaciones residenciales, la vivienda accesible.

Contenidos: Edificios residenciales y multifamiliares, portales de acceso, circulación horizontal, circulación vertical, evacuación, señalización, planteamiento ejercicio de evaluación módulo 6.

Actividades: Clase magistral, conversatorio.

- Semana 12. Accesibilidad en edificaciones residenciales, la vivienda accesible.

Contenidos: La vivienda para toda la vida, acceso, baño, cocina, dormitorios, zonas de estancia, domótica, buenas y malas prácticas de accesibilidad.

Actividades: Clase magistral, conversatorio.

- Semana 13. Accesibilidad en edificaciones residenciales, la vivienda accesible. 
Contenidos: Revisión y seguimiento del ejercicio de evaluación módulo 6.

Actividades: Trabajo de campo, estudio de casos, retroalimentación de lecturas, aplicación normativa, asesoría de diseño, elaboración de textos e infografías.

- Semana 14. Revisión y seguimiento ejercicio de evaluación final.

Actividades: Estudio de casos, aplicación normativa, asesoría de diseño, elaboración de textos e infografías.

- Semana 15. Revisión y seguimiento ejercicio de evaluación final.

Actividades: Estudio de casos, aplicación normativa, asesoría de diseño, elaboración de textos e infografías.

- Semana 16. Entrega ejercicio de evaluación final.

\section{Conclusiones}

Como espacio reflexivo y ejemplarizante para el análisis de la realidad excluyente y saturada de los obstáculos físicos y actitudinales que presenta el entorno construido que habitamos, la cátedra de accesibilidad se manifiesta como un medio eficaz para dar cumplimiento a lo establecido en la Constitución Política colombiana en el capítulo 13, cuando hace referencia a los derechos e igualdad de oportunidades de los ciudadanos.

Ante este principio, el Estado en cumplimiento del mandato constitucional, ha venido desarrollando propuestas como el proyecto para la implementación de la Cátedra de la Paz en todas las instituciones educativas del país, para lo cual se estableció la Ley 1732 de 1 de septiembre de 2014.

En relación con lo anterior, se plantea una extrapolación al ámbito de la accesibilidad, mediante las siguientes conclusiones:
Normatividad técnica incluyente: a partir del ítem "Desarrollo de los talleres" de los resultados de la presente investigación, se proponen los contenidos normativos que ha de abordar la cátedra, para evaluar su pertinencia en los diferentes planes educativos institucionales de las universidades que ofrecen el programa de arquitectura en el país, con el fin de establecer un consenso que defina la metodología que ha de aplicarse para la incorporación del curso de accesibilidad con carácter de obligatoriedad en sus mallas curriculares.

Difusión pertinente y socialización: por medio de entidades como la Asociación Colombiana de Universidades (ASCUN), la Asociación Colombiana de Facultades de Arquitectura (ACFA) y la Sociedad Colombiana de Arquitectos (SCA), entre otras, se puede demarcar un eje de ruta para adelantar las determinaciones necesarias sobre la implementación de la cátedra, propiciando la convocatoria de todos los actores principales involucrados con el tema, para radicar un proyecto de Ley ante los entes de control legislativo pertinentes, que exprese la necesidad de incorporarse la accesibilidad en la formación integral del arquitecto y sus profesiones auxiliares o la posibilidad de su inclusión en eventuales y futras reformas a la educación superior.

Fundamentación y flexibilidad: la cátedra ha de estar reglamentada bajo los principios de la Convención sobre los Derechos de las Personas con Discapacidad aprobada en Colombia por la Ley 1346 de 2009, junto con el marco jurídico y normativo en materia de discapacidad y accesibilidad existente en el país. A su vez, la implementación del plan de asignatura debe adelantarse incorporando los componentes que flexibilicen su ejecución en la modificación de contenidos o actividades curriculares que desarrolle cada institución, sin que se vea afectado el proceso de enseñanza-aprendizaje del estudiante. 
Adaptabilidad en el contexto: la implementación de la cátedra requiere que sea efectuada teniendo en cuenta los contextos locales y regionales donde se desarrolle, incluyendo criterios propios, sistemas constructivos característicos de la población y desarrollos de sostenibilidad o de carácter medioambiental y de eficiencia energética, sin dejar de lado las directrices establecidas en el planteamiento general propuesto.

Estrategias de proyección social: hace referencia a la creación de semilleros o grupos de investigación (Observatorios de la Accesibilidad), que propendan por la generación de planes de accesibilidad a nivel urbano y rural, los cuales generen la integración entre la academia con la sociedad, para la búsqueda de soluciones a las barreras físicas y actitudinales generadas por el hombre, con la posibilidad de gestionar incentivos o subvenciones a través de entidades públicas o privadas comprometidas con el cambio y la innovación social.

\section{Referencias}

Becerra, R. (enero, 2007). Programa Académico Diseño Maxiválido. [Mensaje en un blog]. Recuperado de http:// maxivalidos.blogspot.com.co/2007/01/actividad-desensibilizacin-mi24ene07.html

Beneitone, P., Esquetini, C., González, J., Marty, M., Siufi, G., y Wagenaar, R. (Eds.). (2007). Reflexiones y perspectivas de la educación superior en América Latina. Informe final, Proyecto Tuning América Latina 2004-2007. Universidad de Groningen, Universidad de Deusto. Recuperado de http://tuning.unideusto.org/tuningal/index.php?option=com_docman\&task=view_ca tegory\&catid=22\&Itemid=191\&order=dmdate_ published \&ascdesc $=$ DESC

Castellanos, W. (2015). Accesibilidad universal en todos los programas de arquitectura de universidades colombianas. (Tesis de máster). Universidad de Jaén.
Departamento Administrativo Nacional de Estadística. (2008). Censo general 2005. Nivel nacional. República de Colombia. Recuperado de http://www.dane.gov.co/ censo/files/libroCenso2005nacional.pdf

Fernández, A., y Sarmiento, M. (2015). Diseño inclusivo, cátedra de sede Jorge Eliécer Gaitán. Universidad Nacional de Colombia, sede Bogotá. Recuperado de http://www. facartes.unal.edu.co/postmaster/boletin2/vinculos/diseno_inclusivo.pdf

Ley 361 del Congreso de Colombia. Diario Oficial No. 42978. Bogotá. 7 de febrero de 1997. Recuperado de http:// www.mintic.gov.co/portal/604/articles-3673_documento.pdf

Ley 1346 del Congreso de Colombia. Diario Oficial No. 47427. Bogotá. 31 de julio de 2009. Recuperado de http://www.secretariasenado.gov.co/senado/basedoc/ ley_1346_2009.html

Ley 1732 del Congreso de Colombia. (1 de septiembre, 2014). Cátedra de la Paz. Bogotá. Recuperado de http:// wsp.presidencia.gov.co/Normativa/Leyes/Documents/ LEY\%201732\%20DEL\%2001\%20DE\%20SEPTIEMBRE\%20DE\%202014.pdf

Ley Estatutaria No. 1618 del Congreso de Colombia. Bogotá. 27 de febrero de 2013. Recuperado de http://wsp. presidencia.gov.co/Normativa/Leyes/Documents/2013/ LEY\%201618\%20DEL\%2027\%20DE\%20FEBRERO\%20 DE\%202013.pdf

Ministerio de Salud. (2014). Observatorio Nacional de Discapacidad. Boletín 1. Recuperado de https://www. minsalud.gov.co/sites/rid/Lists/BibliotecaDigital/RIDE/ DE/PS/Boletin-OND-1-2014.pdf

Prieto, M. (2014). Cátedra de paz: Aportes al postconflicto. Boletín de seguimiento legislativo No. 231. Observatorio Legislativo, Instituto de Ciencia Política Hernán Echavarría Olózaga. Recuperado de http://www.icpcolombia. org/archivos/observatorio/boletin_231

Secretaría Jurídica de la Presidencia de la República. (1991). Constitución Política de Colombia de 1991. Recuperado de http://wsp.presidencia.gov.co/Normativa/Documents/Constitucion-Politica-Colombia.pdf

Tejada, C., Tobón, S., Martínez, J., Mendo, C., Moreiro, J., y Ramos, L. (2006). El diseño del plan docente en información y documentación acorde con el espacio europeo de educación superior: un enfoque por competencias. Facultad de Ciencias de la Documentación, Universidad Complutense de Madrid. Recuperado de http://eprints.ucm. es/6005/1/MANUAL.pdf 